\title{
Formaldehyde formation in the glycine cleavage system and its use for an aldolase-based biosynthesis of 1,3- prodanediol
}

\author{
Yingying $\mathrm{Xu}^{1}$, Hao Meng ${ }^{1}$, Jie Ren ${ }^{2}$ and An-Ping Zeng ${ }^{1,3^{*}}$ (D)
}

\begin{abstract}
Glycine cleavage system (GCS) occupies a key position in one-carbon (C1) metabolic pathway and receives great attention for the use of $\mathrm{C} 1$ carbons like formate and $\mathrm{CO}_{2}$ via synthetic biology. In this work, we demonstrate that formaldehyde exists as a substantial byproduct of the GCS reaction cycle. Three causes are identified for its formation. First, the principal one is the decomposition of $N^{5}, N^{10}$-methylene-tetrahydrofolate $\left(5,10-\mathrm{CH}_{2}-\mathrm{THF}\right)$ to form formaldehyde and THF. Increasing the rate of glycine cleavage promotes the formation of 5,10- $\mathrm{CH}_{2}-\mathrm{THF}$, thereby increasing the formaldehyde release rate. Next, formaldehyde can be produced in the GCS even in the absence of THF. The reason is that T-protein of the GCS can degrade methylamine-loaded $\mathrm{H}$-protein $\left(\mathrm{H}_{\text {int }}\right)$ to formaldehyde and ammonia, accompanied with the formation of dihydrolipoyl $\mathrm{H}$-protein $\left(\mathrm{H}_{\text {red }}\right)$, but the reaction rate is less than $0.16 \%$ of that in the presence of THF. Increasing T-protein concentration can speed up the release rate of formaldehyde by $H_{\text {int. }}$ Finally, a certain amount of formaldehyde can be formed in the GCS due to oxidative degradation of THF. Based on a formaldehyde-dependent aldolase, we elaborated a glycine-based one carbon metabolic pathway for the biosynthesis of 1,3-propanediol (1,3-PDO) in vitro. This work provides quantitative data and mechanistic understanding of formaldehyde formation in the GCS and a new biosynthetic pathway of 1,3-PDO.
\end{abstract}

Keywords: Glycine cleavage system, Formaldehyde, 5,10- $\mathrm{CH}_{2}-\mathrm{THF}, 1,3-$ propanediol

\section{Introduction}

Traditional feedstocks for bioproduction processes mainly base on carbohydrates found in food crops, such as simple sugars and starches. However, the global food shortage caused by the rapid growth of the world's population and the continuous reduction of available arable land has stunted the development of the bio-manufacturing industry using these feedstocks [1]. Using lignocelluloses as

\footnotetext{
* Correspondence: aze@tuhh.de

${ }^{1}$ Beijing Advanced Innovation Center for Soft Matter Science and

Engineering, Beijing University of Chemical Technology, North Third Ring

Road 15, Chaoyang District, Beijing 100029, China

${ }^{3}$ Institute of Bioprocess and Biosystems Engineering, Hamburg University of Technology, Denickestrasse 15, D-21073 Hamburg, Germany

Full list of author information is available at the end of the article
}

alternative feedstock in biorefinery has an apparent advantage by not competing with human consumption needs, but still faces many technical challenges, [2]. The utilization of one-carbon (C1) compounds, such as $\mathrm{CO}_{2}$ [3-5], methane [6], methanol [7] and formate [8], has attracted much attention because they are naturally abundant, or available as industrial by-products and are thus cheap for the production of high-value chemicals. Beyond that, the biological assimilation of $\mathrm{C} 1$ compounds for the production of value-added chemicals can help reduce the emission of greenhouse gases such as $\mathrm{CO}_{2}$ and methane which are largely responsible for global warming and climate change [4]. In nature, $\mathrm{C} 1$ assimilation pathway exists in most methylotrophic bacteria [9]. Upon entrance into 
bacterial cells, almost all $\mathrm{C} 1$ compounds such as methanol, methane, formate and dichloromethane are first oxidized or reduced to formaldehyde and subsequently assimilated into the central part of the metabolism of methylotrophic bacteria [10]. Formaldehyde can be further metabolized either via the serine pathway, the ribulose monophosphate (RuMP) pathway or the Calvin-BensonBassham (CBB) cycle in native methylotrophs [11]. Although methylotrophic bacteria can assimilate $\mathrm{C} 1 \mathrm{com}$ pounds, their low specific growth rate is less suitable for large industrial production, and the improvement of natural $\mathrm{C} 1$ metabolic pathways in native hosts is often difficult due to lack of efficient genetic tools [12]. Hence, it is desirable to introduce synthetic $\mathrm{C} 1$ assimilation pathways to model microorganisms such as Saccharomyces cerevisiae and Escherichia coli, which have been extensively exploited in the biotechnology industry [13]. Recent studies have demonstrated that a wide variety of non-native metabolic pathways can be integrated into these model microorganisms, allowing the synthesis of value-added chemicals, such as 1,3-propanediol (1,3-PDO). At present, the major 1,3-PDO biosynthesis pathways include the routes "glycerol to 1,3-PDO", "glucose to 1,3-PDO via glycerol" and "glucose to 1,3-PDO via homoserine" [14]. Our recent study [15] showed a novel pyruvate-based $\mathrm{C} 1$ metabolic pathway to synthesize 1,3-PDO from formaldehyde and glucose. It was successfully implemented in E. coli and demonstrated that $\mathrm{C} 1$ compounds like methanol can be used to synthesize 1,3-PDO via formaldehyde as a metabolic intermediate. The incorporation of $\mathrm{C} 1 \mathrm{com}-$ pounds into the formation of 1,3-PDO synthesis opens up the possibility of incorporating $\mathrm{CO}_{2}$ into the production of bulk-chemicals like 1,3-PDO since formaldehyde and methanol can be generated from $\mathrm{CO}_{2}$ electrochemically. However, formaldehyde as a substrate suffers from the problem of toxicity to cell growth. Although methanol is less toxic to most microorganisms, the reaction catalyzed by methanol dehydrogenase is thermodynamic unfavorable and limits the conversion rate of this pathway. One of the purposes of the present work is to explore the possibility of generating formaldehyde from glycine using the glycine cleavage system (GCS) for 1,3-PDO synthesis. This may help to circumvent critical issues like substrate toxicity and limited conversion rate in the direct use of formaldehyde and/or methanol. GCS is widely present in the mitochondria of plant and animal tissues as well as in the cytosol of most bacteria. It consists of four different component proteins named as P-protein (glycine decarboxylase; EC 1.4.4.2), T-protein (aminomethyltransferase; EC 2.1.2.10), L-protein (dihydrolipoyl dehydrogenase; EC 1.8.1.4) and H-protein (lipoamide-containing aminomethylene carrier) and catalyzes the oxidative decarboxylation and deamination of glycine to yield one molecule each of $\mathrm{CO}_{2}$ and ammonia, in accompany with the transfer of a methylene group to tetrahydrofolate (THF), forming thereby $5,10-\mathrm{CH}_{2}$-THF (Eq. 1):

$$
\begin{aligned}
& \text { Glycine }+\mathrm{NAD}^{+}+\mathrm{THF} \leftrightarrow \mathrm{CO}_{2}+\mathrm{NH}_{3} \\
& +5,10-\mathrm{CH}_{2}-\mathrm{THF}+\mathrm{NADH}+\mathrm{H}^{+}
\end{aligned}
$$

In the enzymatic reaction cycle of the GCS (Fig. 1), lipoylated $\mathrm{H}$-protein plays a pivotal role by acting as a mobile substrate, which interacts with the three other proteins via its freely swinging lipoyl arm. The degradation of glycine molecules is first triggered by P-protein to yield $\mathrm{CO}_{2}$ and methylamine-loaded $\mathrm{H}$-protein $\left(\mathrm{H}_{\text {int }}\right)$. $\mathrm{H}_{\text {int }}$ then forms a complex with T-protein, leading to a degradation of the aminomethyl moiety to ammonia and 5,10- $\mathrm{CH}_{2}$-THF in the presence of THF, whereas $\mathrm{H}_{\text {int }}$ leaves as dihydrolipoyl $\mathrm{H}$-protein $\left(\mathrm{H}_{\mathrm{red}}\right)$ in a reduced form. After that $\mathrm{H}_{\text {red }}$ is oxidized to the oxidized form of $\mathrm{H}$-protein $\left(\mathrm{H}_{\mathrm{ox}}\right)$ under the catalysis of the L-protein and the accompanying conversion of $\mathrm{NAD}^{+}$to NADH [16]. The formation of NADH is usually used for assay of the GCS reaction rate.

Kikuchi et al. [17] discovered formaldehyde formation in the GCS. Guilhaudis et al. [18] reported the decomposition of $\mathrm{H}_{\text {int }}$ to $\mathrm{H}_{\text {red }}$ and formaldehyde in the absence of THF. However, till now, no systematic studies have been carried out on the formation of formaldehyde in the GCS and the reasons for its formation are not yet clear. In this study, we carried out a systematic and quantitative study and first revealed three possible sources of formaldehyde formation in the GCS. Furthermore, we demonstrated that formaldehyde generated from glycine via the GCS is able to be directly used for the synthesis of 1,3-PDO through our recently proposed aldolase-based metabolic pathway [15]. It is noted that although glycine is a C2 compound used here for 1,3PDO synthesis, the study of this new pathway could give some useful hints for the utilization of $\mathrm{C} 1$ compounds such as formate. Similar to the glycine utilization via GCS to generate 5,10-CH2-THF (Fig. 1), formate can be catalyzed by formate tetrahydrofolate ligase and the bifunctional methenyltetrahydrofolate cyclohydrolase/ dehydrogenase to generate $5,10-\mathrm{CH} 2-\mathrm{THF}$ which is decomposed to formaldehyde and thus also used for the synthesis of 1,3-PDO.

\section{Materials and methods Materials}

All chemicals were of reagent grade and purchased from commercial suppliers including Aladdin (Shanghai, China), Yuanye Bio-Technology (Shanghai, China), Sigma-Aldrich (St. Louis, MO, USA), unless otherwise noted. Phusion High-Fidelity DNA polymerase and restriction enzymes were purchased from Thermo-Fisher Scientific (Pittsburgh, PA, USA). T4 DNA ligase was 


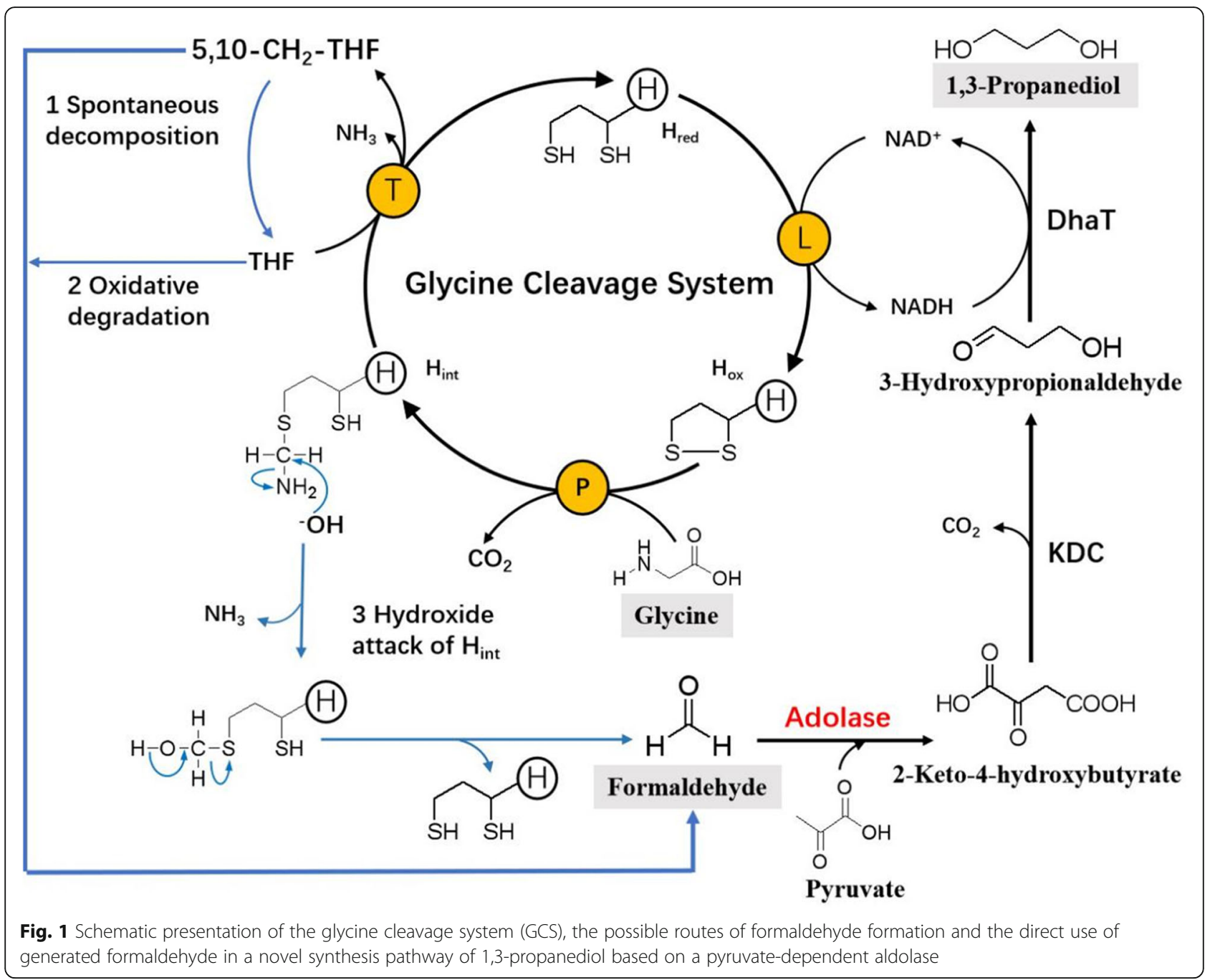

purchased from New England Biolabs (Ipswich, MA, USA). DNA extraction kit and gel extraction kit were purchased from Promega (Madison, WI, USA). Oligonucleotide primers were ordered from GeneWiz (Suzhou, China). $\mathrm{Ni}^{2+}$-NTA resin was purchased from Genscript (Nanjing, China). Amicao ${ }^{\circ}$ Ultra-15 filtration devices (molecular cutoff 10-100 KDa) were purchased from Millipore (Billerica, MA, USA). BCA protein assay kit and precast-gel for sodium dodecyl sulfate-polyacrylamide gel electrophoresis (SDS-PAGE) were purchase from Solar Bio (Beijing, China).

Plasmid construction, protein expression and purification Genes encoding P-protein, T-protein, H-protein, Lprotein and SHMT (serine hydroxymethyltransferase) were amplified from the genomic DNA of E. coli K12 and cloned into corresponding expression vectors (Table 1). E. coli BL21 cells were used as the host for protein overexpression and purification. The recombinant strains harboring pET-P, pET-T, pET-L, pET-H and
pET-SHMT, respectively, were grown at $37^{\circ} \mathrm{C}$ in LBmedium supplemented with an appropriate antibiotic $(100 \mu \mathrm{g} / \mathrm{mL}$ ampicillin or $50 \mu \mathrm{g} / \mathrm{mL}$ kanamycin) until the $\mathrm{OD}_{600}$ reached 0.8 , and gene expression was then induced by adding $0.2 \mathrm{mM}$ IPTG for additional $12 \mathrm{~h}$ at $30{ }^{\circ} \mathrm{C}$. From each culture, cells were collected by centrifugation at $3500 \mathrm{rpm}$ for $10 \mathrm{~min}$, resuspended in $50 \mathrm{mM}$ Tris- $\mathrm{HCl}$ ( $\mathrm{pH} 7.5)$, and disrupted by sonication. Cell debris was removed by centrifugation at $100,000 \mathrm{rpm}$ for 1 $h$, and the supernatant was purified using a $\mathrm{Ni}^{2+}-\mathrm{NTA}$ column to obtain purified enzymes for activity assays. Purified proteins were checked by SDS-PAGE and protein concentrations were quantified using $\mathrm{BCA}$ protein assay kit.

\section{Glycine cleavage system activity assay}

The standard reaction mixture contained Tris- $\mathrm{HCl}(50$ $\mathrm{mM}, \mathrm{pH} 7.5), 0.5 \mathrm{mM}$ THF, $20 \mathrm{mM} \beta$-mercaptoethanol (MCE), $25 \mu \mathrm{M}$ pyridoxal 5 '-phosphate (PLP), $5 \mathrm{mM}$ $\mathrm{NAD}^{+}, 5 \mu \mathrm{M}$ P-protein, $5 \mu \mathrm{M}$ T-protein, $5 \mu \mathrm{M} \mathrm{L}$-protein 
Table 1 Strains and plasmids used for this study

\begin{tabular}{lll}
\hline & Description & Reference \\
\hline E. coli Strains & & WEIDI Ltd. \\
Top 10 & Host for cloning plasmids & WEIDI Ltd. \\
BL21 (DE3) & Host for protein overexpression and purification & Novagen \\
Plasmids & & Novagen \\
pET28a (+) & Plasmid for protein overexpression & This study \\
pET22b (+) & Plasmid for protein overexpression & This study \\
pET28a-P & pET28a vector containing P-protein gene (NCBI No. WP_112929453.1) & This study \\
pET28a-T & pET28a vector containing T-protein gene (NCBI No. WP_099356926.1) & This study \\
pET28a-H & pET28a vector containing H-protein gene (NCBI No. WP_001295377.1) & This study \\
pET28a-L & pET28a vector containing L-protein gene (NCBI No. WP_110826218.1) & Laboratory stock [15] \\
pET22b-SHMT & pET22b vector containing SHMT gene (NCBI No. WP_072756891.1) & \\
pRSFduet-1-KHB-KDC-DhaT & pRSFduet-1 vector containing KHG gene (NCBI No. WP_000800512), KDC & gene (NCBI No. WP_046124870) and DhaT gene (NCBI No. WP_004900746) \\
\hline
\end{tabular}

and $10 \mu \mathrm{M} \mathrm{H}$-protein. After premixing and centrifugation, reactions were initiated by the addition of $50 \mathrm{mM}$ glycine. Glycine degradation was determined at $37^{\circ} \mathrm{C}$ by measuring NADH formation using an Enspire multimode plate reader (PerkinElmer, USA) as described previously $[19,20]$.

\section{Inhibitory effect of formaldehyde on the activity of the GCS}

The investigation of formaldehyde toxicity was performed by adding various concentrations of formaldehyde to the GCS reaction mixture to determine the initial rates of the GCS. The composition of the standard reaction mixture was the same as described in 2.3. After premixing and centrifugation, reactions were initiated by the addition of $50 \mathrm{mM}$ glycine and different concentration of formaldehyde $(0-5 \mathrm{mM})$. The initial rates of $\mathrm{NADH}$ formation in different concentrations of formaldehyde samples were used to assess the effect of formaldehyde toxicity.

\section{1,3-PDO synthesis}

The three-enzyme (KHG, KDC, and DhaT) reaction cascade for the synthesis of 1,3-PDO was assayed as described previously [15]. The proof-of-concept biosynthesis of 1,3-PDO was conducted by mixing purified GCS enzymes $(5 \mu \mathrm{M}$ P-protein, $5 \mu \mathrm{M}$ T-protein, $5 \mu \mathrm{M} \mathrm{L}$-protein and $10 \mu \mathrm{M}$ H-protein) and a crude cell extract (containing KHG, KDC, and DhaT) with $50 \mathrm{mM}$ Tris- $\mathrm{HCl}$ buffer (pH 7.5), $0.5 \mathrm{mM}$ THF, $20 \mathrm{mM} \beta$-mercaptoethanol, $25 \mu \mathrm{M}$ PLP, $1 \mathrm{mM} \mathrm{NAD}+10 \mathrm{mM}$ sodium pyruvate, and $1 \mathrm{mM}$ thiamine pyrophosphate (TPP). The reaction was started by adding $10 \mathrm{mM}$ glycine, kept at $37^{\circ} \mathrm{C}$ for $7 \mathrm{~h}$, and analyzed by GC-MS.

\section{Analytical methods}

Free formaldehyde in GCS reaction mixtures was derivatized with 2,4-dinitrophenylhydrazine (2,4-DNPH) to form 2,4-dinitrophenylhydrazone, before quantified by HPLC (Fig. 2). To this end, $0.2 \mathrm{~mL}$ of a reaction mixture was mixed with $0.2 \mathrm{~mL}$ of $10 \%(\mathrm{v} / \mathrm{v})$ trifluoroacetic acid (TFA), $0.1 \mathrm{~mL}$ of 2,4-DNPH $(1 \mathrm{~g} / \mathrm{L})$ and $0.5 \mathrm{~mL}$ of acetonitrile. Derivatization occurred at $40{ }^{\circ} \mathrm{C}$ for $30 \mathrm{~min}$. The 2,4-dinitrophenylhydrazone derivative was analyzed using a Wondasil $\mathrm{C}_{18}$ column $(4.6 \mathrm{~mm} \times 150 \mathrm{~mm}, 5 \mu \mathrm{m}$, Shimadzu, Japan) with acetonitrile/water $(50,50)$ containing $0.095 \%$ TFA as the mobile phase at a flow rate of $1.0 \mathrm{~mL} / \mathrm{min}$, and detected at the wavelength of $352 \mathrm{~nm}$.

1,3-PDO was analyzed by GC-MS as described in Wang et al. using a QP2020 system (Shimadzu, Japan) equipped with a SH-Rxi-5Sil-MS column (Shimadzu, Japan), with helium as the carrier gas. The oven temperature was programmed to be held at $100^{\circ} \mathrm{C}$ for 2 min, raised at a gradient of $15^{\circ} \mathrm{C} \mathrm{min}^{-1}$ to $270^{\circ} \mathrm{C}$ and held for $12 \mathrm{~min}$ at $270{ }^{\circ} \mathrm{C}$.

\section{Results and discussion}

\section{Formaldehyde as a by-product in the GCS reaction}

In our initial study of the GCS as a potentially important route for formate-based biosynthesis, a series of experiments at different concentrations of glycine, $\mathrm{H}_{\mathrm{ox}}, \mathrm{NAD}^{+}$ and THF were carried out and the NADH production was monitored as a measure of the reaction rate of the GCS. As shown in Fig. 3, while increasing the concentration of glycine, $\mathrm{H}_{\mathrm{ox}}$ or $\mathrm{NAD}^{+}$the initial reaction rate increased gradually, but in the case of THF, the reaction first showed a strong acceleration when the concentration of THF added increased from 0.1 to $0.5 \mathrm{mM}$, and then stagnation when the concentration of THF was further raised to $0.8 \mathrm{mM}$, and even decrease when $2 \mathrm{mM}$ THF was used. According to a previous study [21], high 

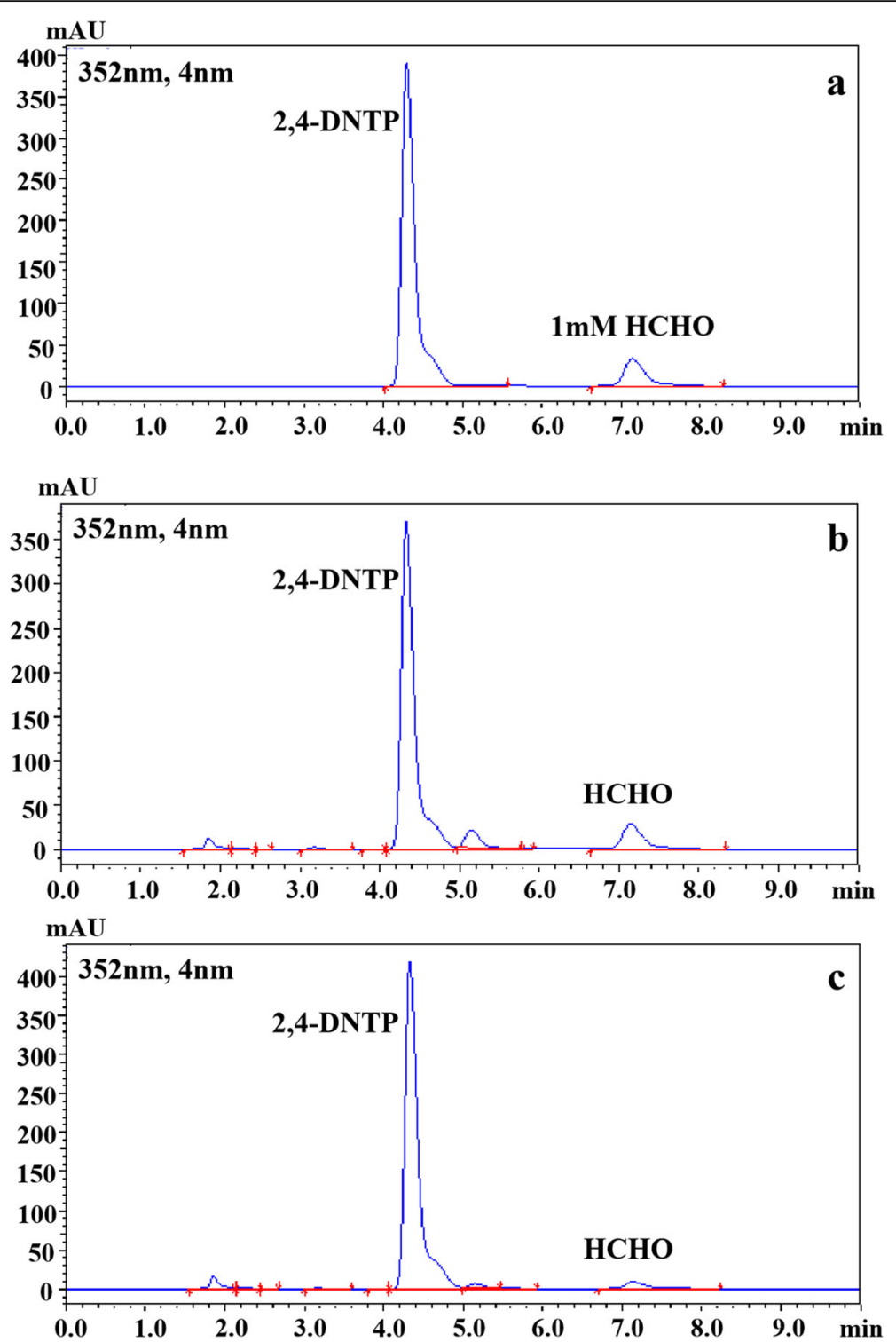

Fig. $\mathbf{2 H P L C}$ analysis of formaldehyde from different samples. a $1 \mathrm{mM}$ formaldehyde standard. $\mathbf{b}$ GCS reaction mixture after 2 h. c GCS reaction mixture without adding GCS enzymes after $2 \mathrm{~h}$

concentrations of THF can inhibit the conversion of glycine to $\mathrm{CO}_{2}$ catalyzed by P-protein, which may be the reason for the results shown in Fig. 3d. According to Eq. 1 the formation of NADH is stoichiometrically linked to the consumption of THF and the corresponding formation of 5,10- $\mathrm{CH}_{2}$-THF from the methylene carbon unit of glycine and THF. Unexpectedly, the production of NADH exceeded the added amount of THF $(0.5 \mathrm{mM})$ in Figs. 3a-c, when the initially added concentrations of the specific substrates in Figs. 3a-c increased above certain levels. This was also observed in Fig. 3d except for the case with $2 \mathrm{mM}$ THF, where a reaction inhibition by THF was obvious. For the stoichiometric discrepancy between the formation of NADH and the consumption of THF a possible explanation would be that $5,10-\mathrm{CH}_{2}$ THF formed in the GCS reaction is highly instable and can be spontaneously decomposed into formaldehyde and THF under the given experimental conditions $\mathrm{pH}$ 7.5) [22]. THF then enters again into the GCS reaction, leading to the further conversion of glycine and the formation of additional NADH.

To verify the supposition, an assay method to determine free formaldehyde using HPLC was established, with a pre-column derivation of formaldehyde with 2,4dinitrophenylhydrazine. The results of HPLC (Fig. 2) showed that formaldehyde was indeed formed in the reaction samples. Thus, it is proved that formaldehyde is one product of the in vitro GCS reaction in this study. 

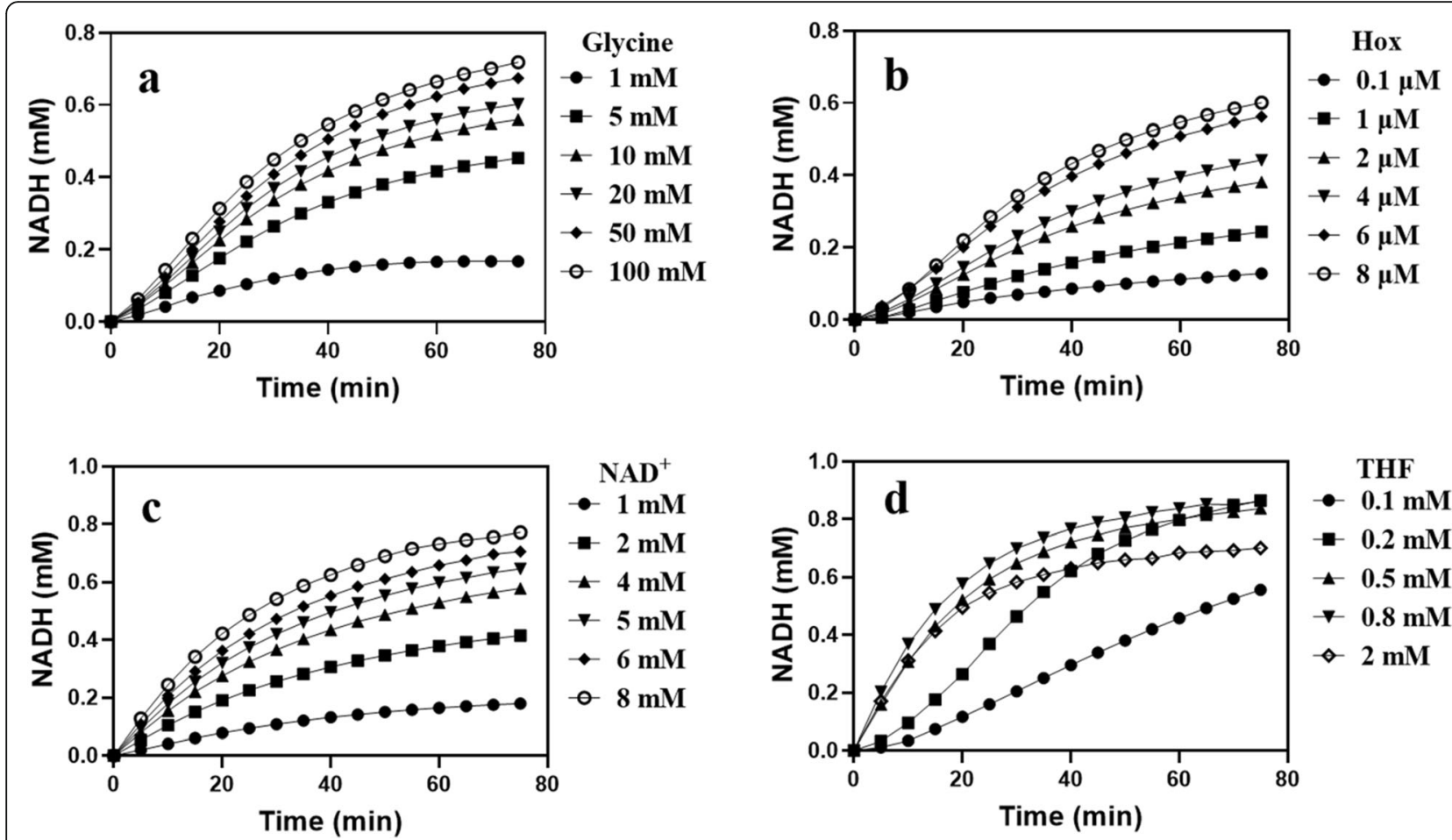

Fig. 3 Effects of concentrations of different substrates on the overall GCS reaction rate as determined by the time course of NADH production in the presence of $\mathbf{a}$ and $\mathbf{b} 0.2 \mu \mathrm{M}$ P-protein, $\mathbf{c} 0.2 \mu \mathrm{M}$ L-protein and $\mathbf{d} 0.2 \mu \mathrm{M}$ T-protein. THF in $\mathbf{a}, \mathbf{b}$ and $\mathbf{c}$ was $0.5 \mathrm{mM}$. Other reaction components and GCS enzymes are present in excess as specified in Materials and Methods

It should be mentioned that formaldehyde was also detected in the reaction mixture free of GCS enzymes after $2 \mathrm{~h}$, as shown in Fig. 2c. To find out the reason for the existence of formaldehyde in the enzyme-free reaction mixture, we determined the formaldehyde concentrations in solutions of the different substrates of the GCS reaction mixture, respectively. The results revealed the presence of a small amount of formaldehyde only in the THF solution $(0.06 \mathrm{mM}$ formaldehyde in $0.5 \mathrm{mM}$ THF solution). As indicated in literature [23], THF decomposition through oxidative degradation may release formaldehyde as well (Fig. 4a). To systematically examine THF stability with regard to formaldehyde formation, THF solutions containing different concentrations of dithiothreitol (DTT) as antioxidant was placed in the air at $37^{\circ} \mathrm{C}$. Figure $4 \mathrm{~b}$ shows that exposure to air resulted in the oxidative decomposition of THF to formaldehyde, but the amount of formaldehyde released from THF decreased with increased concentration of DTT. Comparing three commonly used antioxidants, ascorbate has the strongest ability to prevent the release of formaldehyde by THF degradation, followed by MCE and DTT (Fig. 4c). The result in Fig. 4d demonstrates that regarding formaldehyde formation THF was also susceptible to acidic conditions and the release of formaldehyde increased with increased acidity.
Factors affecting formaldehyde formation in the GCS reaction

We sought to assess the effects of key factors affecting the GCS reaction activity and formaldehyde formation. Similar to the control group without adding GCS enzymes (without enzyme) and in contrast to the normal group, there was almost no NADH formation in the experimental group without adding THF (without THF) (Fig. 5a). Therefore, THF plays an indispensable role in initiating and accelerating the GCS reaction rate. Interestingly, in the absence of THF, T-protein was still able to cause a change in the overall conformation of the $\mathrm{H}_{\mathrm{int}}$ (methylaminated form), leading to the release of the lipoamide methylamine arm from the cleft at the surface of the H-protein. According to Guilhaudis et al. [18] this situation favors nucleophilic attack by $\mathrm{OH}^{-}$of the carbon atom in the aminomethyl group. Such an attack can lead to a slow release of $\mathrm{NH}_{3}$ and formaldehyde, which is accompanied by the full reduction of the lipoamide arm (Fig. 1). As shown in Fig. 5b, T-protein catalyzed the release of ammonia and formaldehyde from $\mathrm{H}_{\text {int }}$ in the absence of THF, and the reaction rate increased with increased concentration of T-protein. However, at the same concentration of $\mathrm{T}$-protein $(5 \mu \mathrm{M})$, the reaction rate of $\mathrm{T}$-protein catalyzed release of formaldehyde $(0.08 \mu \mathrm{M} / \mathrm{min})$ was less than $0.16 \%$ of the formaldehyde 

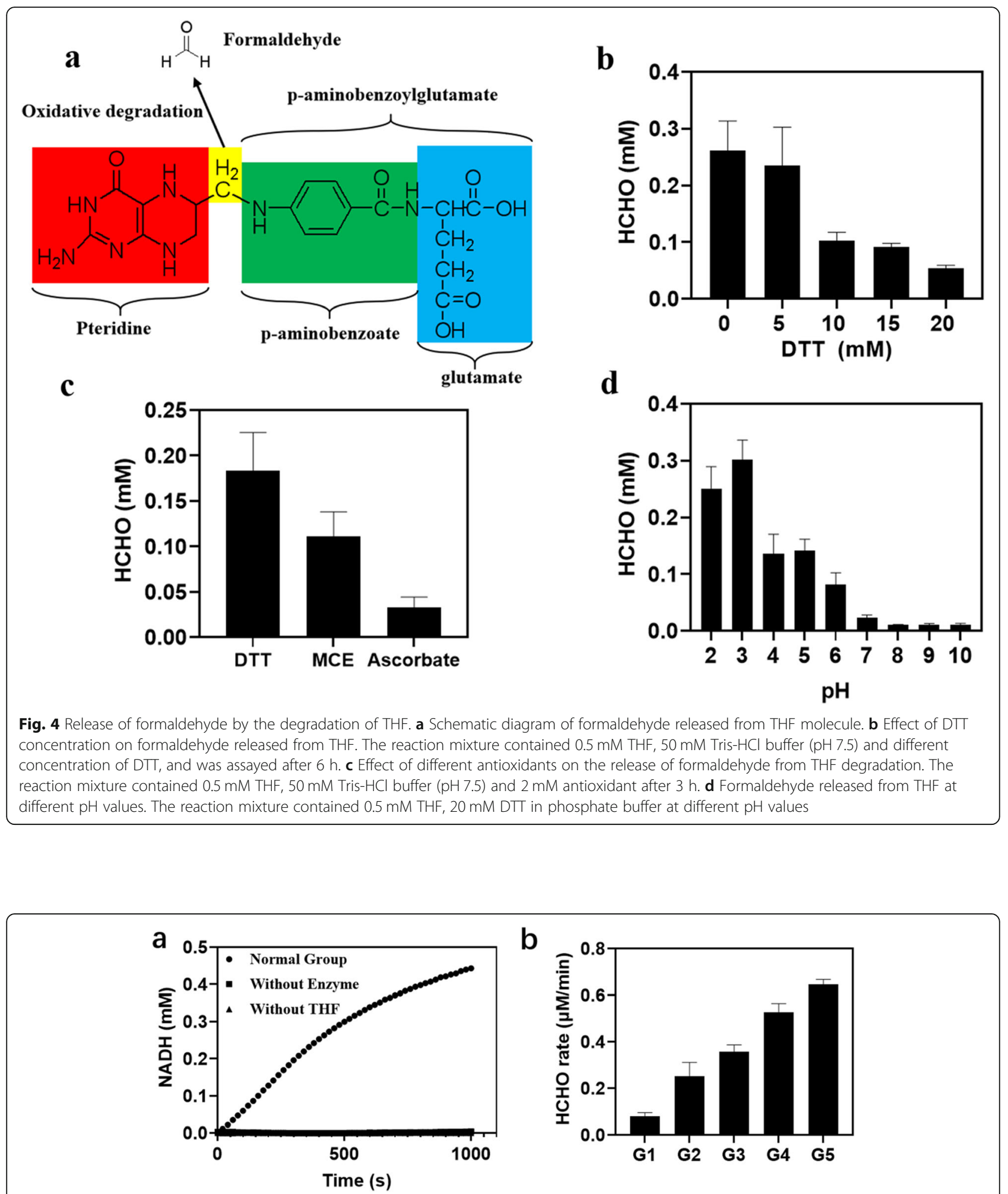

b

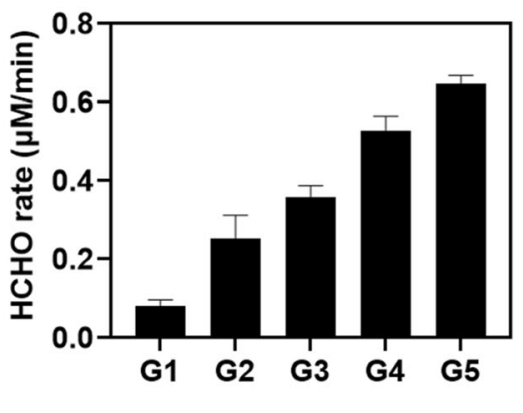

Fig. 5 a GCS activity assay confirming the necessity of THF for the operation of the whole system. "Normal Group" refers to a GCS reaction mixture as specified in the Materials and Methods section without missing any reaction components and enzymes. "Without Enzyme" refers to a reaction mixture containing the same concentrations of substrates except that no GCS enzymes were added. "Without THF" refers to a reaction mixture containing all reaction components and enzymes except for THF. b Formaldehyde formation in the overall GCS reaction catalyzed by Tprotein at concentrations of 5, 10, 25, 50, and $80 \mu \mathrm{M}$ from G1 to G5, respectively, after $5 \mathrm{~h}$. Other reaction components and enzymes were the same as described in the Materials and Methods section 
formation rate $(50 \mu \mathrm{M} / \mathrm{min})$ measured in the presence of THF. Thus, in the absence of THF, the methylamine transfer reaction rate catalyzed by $\mathrm{T}$-protein is extremely low, which is not the primary cause of formaldehyde formation in the GCS reaction system. A similar suggestion was given by Kikuchi et al. [17] but without providing any quantitative data.

Margaretha et al [22] reported that 5,10- $\mathrm{CH}_{2}$-THF dissociates into THF and formaldehyde at physiological $\mathrm{pH}$ and acidic $\mathrm{pH}$. In fact, $5,10-\mathrm{CH}_{2}$-THF is only stable towards dissociation into THF at $\mathrm{pH}>8$ or in the presence of a huge molar excess of formaldehyde. Since the GCS reaction mixture used in the present work was buffered at $\mathrm{pH} 7.5,5,10-\mathrm{CH}_{2}$-THF was more easily decomposed into formaldehyde and THF. The generated THF reentered the reaction so that the overall GCS reaction proceeded to an extent, leading to higher NADH production than stoichiometrically expected. In order to further prove our conjecture, we constructed in vitro a GCS-SHMT cascade reaction system to timely remove the GCS-catalyzed product 5,10-CH2-THF. Serine hydroxymethyltransferase (SHMT; EC 2.1.2.1) is a pyridoxal 5'-phosphate (PLP) dependent enzyme that catalyzes the reversible conversion of $5,10-\mathrm{CH}_{2}-\mathrm{THF}$ and glycine to THF and serine and therefore closely associated with the function of the GCS in C1 metabolic pathways. The results in Fig. 6 show that compared with the continuous increase in the concentration of formaldehyde in the R1 group (containing only GCS enzymes) over time, the concentration of formaldehyde in the R2 group (containing SHMT in addition to the GCS enzymes) did not increase with time and remained at almost zero, indicating that most formaldehyde formed in the GCS reaction was from decomposition of $5,10-\mathrm{CH}_{2}$-THF.

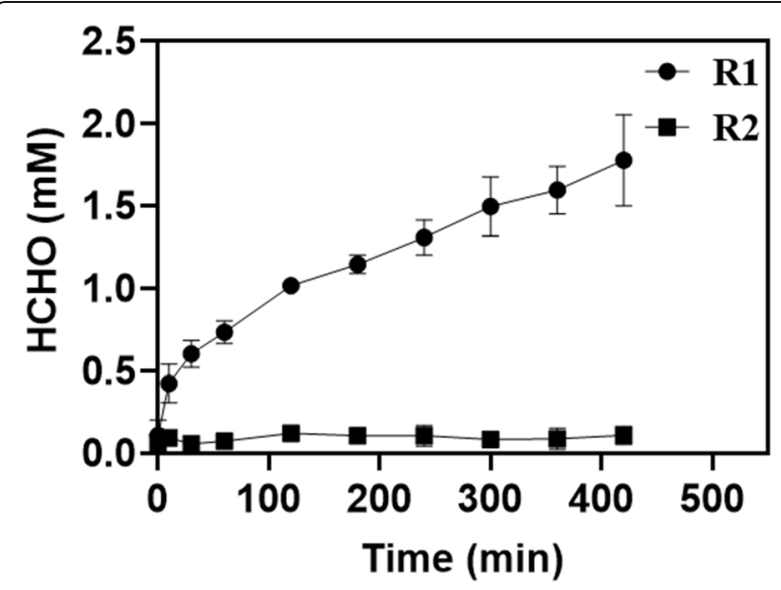

Fig. 6 Effect of SHMT on formaldehyde production in the GCS reaction. $\mathrm{R} 1$ indicates a GCS reaction mixture as specified in the Materials and Methods section. R2 refers to a reaction mixture similar to R1 with the addition of SHMT
We further observed that concentrations of substrates not only affected the overall reaction rate of the GCS (Fig. 3), but also determined the rate of formaldehyde formation, as shown in Fig. 7. The overall reaction rate of GCS increased gradually as the substrate concentration (either glycine, $\mathrm{H}_{\mathrm{ox}}$, or $\mathrm{NAD}^{+}$) was increased (Fig. 3), and increasing the rate of glycine cleavage promoted the formation of $5,10-\mathrm{CH}_{2}-$ THF, thereby increasing the formaldehyde release rate. In the case of THF increased concentration led first to higher formaldehyde generation rate, however, further increase in the concentration of THF resulted in decrease in the rate of formaldehyde formation. This can be explained by the inhibition behavior of a high concentration of THF both on the overall GCS reaction and on the dissociation of $5,10-\mathrm{CH}_{2}$-THF (Fig. 3d). To better understand the relationship between the concentration of THF and the rate of formaldehyde formation, the construction of a GCS kinetic model is desirable.

Concentrations of substrates added into the reaction mixture also affected the final yield of formaldehyde. According to the experimental results in Fig. 7, when the initial concentration of a substrate (either glycine, $\mathrm{H}_{\mathrm{ox}}$, or $\mathrm{NAD}^{+}$) was increased, the final concentration of formaldehyde generated increased as well. However, when the initial substrate concentration was above a certain level, the final concentration of formaldehyde reached did not further increase with increased substrate concentration but remained nearly constant. This may be due to the toxicity of formaldehyde which is significant at a concentration above 3-4 mM (data not shown). In the case of THF as shown in Fig. 7d, even at relatively low THF concentrations, the formation of formaldehyde in the GCS reaction was already significant.

\section{1,3-PDO formation from formaldehyde via glycine}

In a previous work of our research group, we successfully used a pyruvate-dependent aldolase to condense formaldehyde and pyruvate into 2-keto-4-hydroxybutyrate (HOBA), and utilized two further enzymes, a branchedchain alpha-keto acid decarboxylase (KDC) and a NADHdependent 1,3-PDO oxidoreductase (DhaT), to convert HOBA into 1,3-PDO. In the present work, instead of directly using formaldehyde as a substrate, we utilized glycine as a starting material and formaldehyde dissociated from $5,10-\mathrm{CH}_{2}$-THF, which is generated in the GCS reaction cycle, to synthesize 1,3-PDO (Fig. 1). As shown in Fig. 8, 1,3-PDO was indeed in vitro synthesized in such an enzyme cascade reaction system with glycine and pyruvate as substrates. Slightly higher 1,3-PDO concentration was achieved in comparison with the direct use of formaldehyde and pyruvate. The use of glycine has the following advantages: (1) reduced toxicity towards the enzymes used 

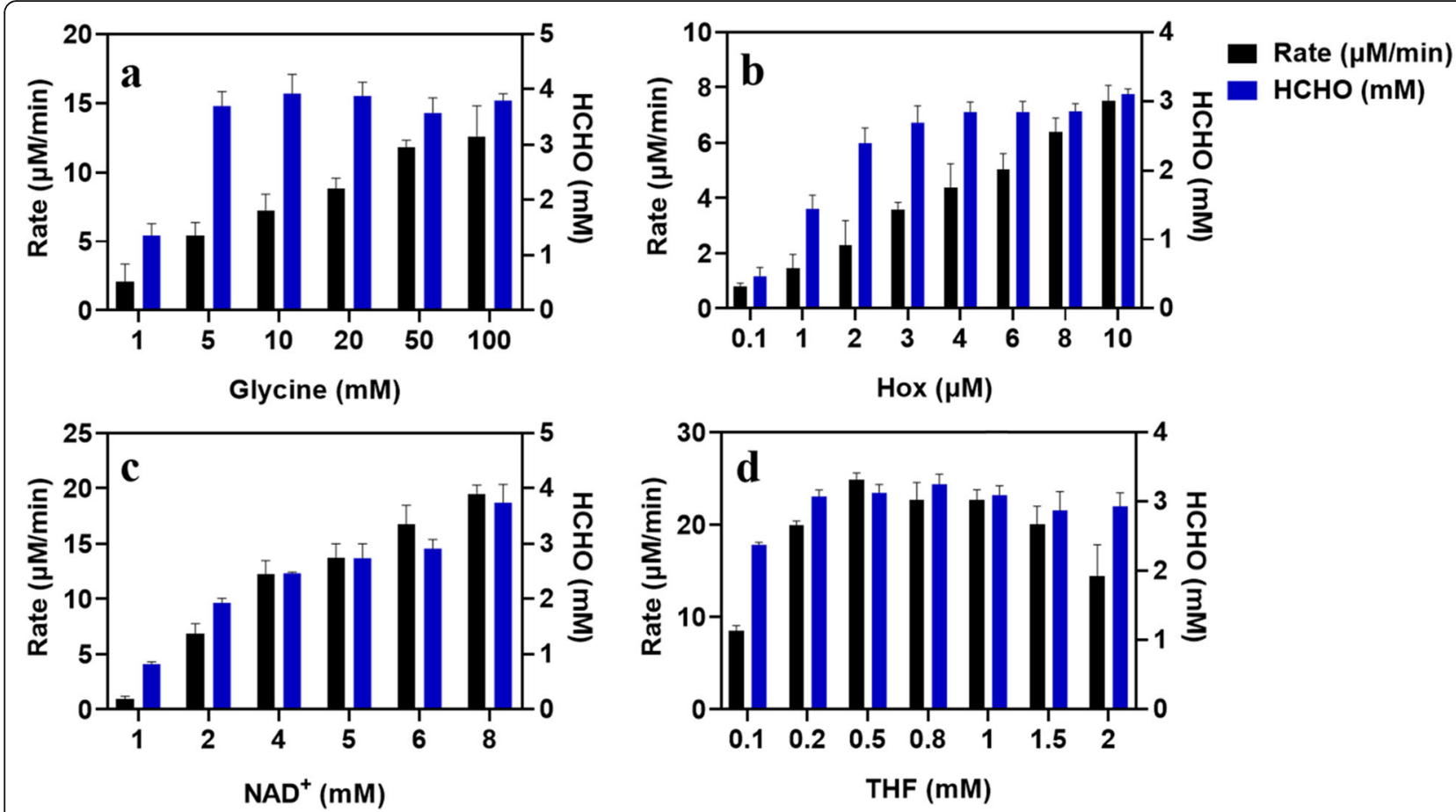

Fig. 7 Effects of concentrations of different substrates on the formation rate and final concentration of formaldehyde in the GCS reaction. The compositions of the reaction mixtures were as specified in the Materials and Methods section, except for that in $\mathbf{a}$ and $\mathbf{b}$ P-protein added was $0.2 \mu \mathrm{M}$, in c T-protein added was $0.2 \mu \mathrm{M}$, and in $\mathbf{d} \mathrm{L}$-protein added was $0.2 \mu \mathrm{M}$

compared to the direct use of formaldehyde; and (2) NADH produced by the GCS can be utilized for the reduction of 3-HPA to 1,3-PDO catalyzed by DhaT. It is known that the yield of 1,3-PDO is mainly limited by the availability of reducing power in form of NADH or NADPH.

It should be mentioned that the possibility of using formaldehyde from the GCS reaction cycle for in vivo 1, 3-PDO biosynthesis should be experimentally checked carefully, since $5,10-\mathrm{CH}_{2}$-THF is involved in many bioreactions in vivo [24]. Furthermore, the intracellular

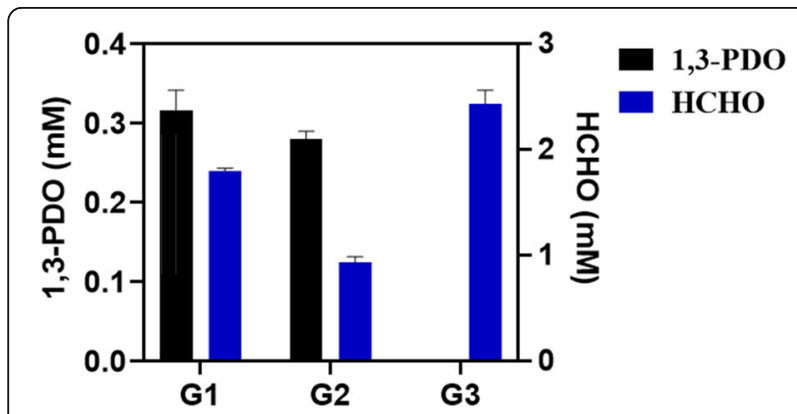

Fig. 8 In vitro 1,3-PDO synthesis. G1: glycine and pyruvate as substrates via GCS coupled with the enzymes KHG, KDC, and DhaT; G2: formaldehyde ( $5 \mathrm{mM})$ and pyruvate as substrates with only the three enzymes KHG, KDC, and DhaT; G3: glycine as substrate catalyzed by GCS without adding KHG, KDC, and DhaT environment conditions should be considered. As mentioned above the existence of SHMT may prevent the accumulation of $5,10-\mathrm{CH}_{2}$ - THF and thus the release of formaldehyde. Irrespective of the possibility of using formaldehyde from the GCS reaction cycle for biosynthesis this work provided useful quantitative data and mechanistic understanding of formaldehyde formation in the GCS reaction. Most of previous studies focus on the catalytic mechanism of each components in GCS, and few results were published regarding the kinetic properties of the overall reaction. In fact, a convenient way to better understand the kinetic behavior of GCS and the synergistic effect of each components is to study them as a whole. Compared with previous work, our systematic study on GCS revealed new and quantitative information about glycine-derived formaldehyde formation. Although 5,10- $\mathrm{CH}_{2}$-THF is known to dissociate into formaldehyde and THF in vitro, the formation of formaldehyde in GCS under the presence of THF is first quantitatively studied in this work. We also confirmed the significance of THF in promoting the overall GCS reaction, and studied the factors affection the oxidative degradation of THF to release formaldehyde. Importantly, the results clearly showed that the measurement of NADH is not a reliable method to study the kinetics of GCS reactions since its formation rate is affected by the availability of THF. The latter is involved in two of the three mechanisms of 
formaldehyde formation related to the GCS system which were examined in this work. As recently pointed out by Hong et al [24] it is desired to develop more efficient and reliable analytic methods for direct array of the individual reaction steps of the GCS system.

\section{Conclusion}

In this work, we studied the kinetic behavior of the GCS and proved that a substantial amount of formaldehyde was formed in the GCS reaction cycle. Three sources of formaldehyde formation were identified. It was shown that the main reason of formaldehyde formation was caused by the dissociation of $5,10-\mathrm{CH}_{2}$-THF under the given experimental conditions. The formation of formaldehyde in GCS should be carefully considered in studying GCS and using it for C1-based synthetic biology. Combining the findings from this work with previous research results of our lab, we constructed a novel 1,3PDO biosynthetic pathway with glycine and pyruvate as substrates, and conceptually proved the feasibility of this pathway in vitro.

\section{Acknowledgments}

This work is financially supported by the Beijing Advanced Innovation Center for Soft Matter Science and Engineering.

\section{Authors' contributions}

AZE supervised this study. AZE, JR and YYX conceived and designed the experiments. YYX and HM performed the experiments. AZE, JR and YYX discussed the data and wrote the manuscript. The author(s) read and approved the final manuscript.

\section{Availability of data and materials}

Please contact the corresponding author for data requests.

\section{Ethics approval and consent to participate}

Not applicable.

\section{Consent for publication}

Not applicable.

\section{Competing interests}

The authors declare that they have no competing interests.

\begin{abstract}
Author details
${ }^{1}$ Beijing Advanced Innovation Center for Soft Matter Science and Engineering, Beijing University of Chemical Technology, North Third Ring Road 15, Chaoyang District, Beijing 100029, China. ${ }^{2}$ State Key Laboratory for Biology of Plant Diseases and Insect Pests/Key Laboratory of Control of Biological Hazard Factors (Plant Origin) for Agri-product Quality and Safety, Ministry of Agriculture, Institute of Plant Protection, Chinese Academy of Agricultural Sciences, Beijing 100081, China. ${ }^{3}$ Institute of Bioprocess and Biosystems Engineering, Hamburg University of Technology, Denickestrasse 15, D-21073 Hamburg, Germany.
\end{abstract}

Received: 24 February 2020 Accepted: 24 April 2020

Published online: 14 May 2020

\section{References}

1. Hadjipaschalis I, Poullikkas A, Efthimiou V. Overview of current and future energy storage technologies for electric power applications. Renew Sust Energ Rev. 2009;13:1513-22.

2. Menon V, Rao M. Trends in bioconversion of lignocellulose: biofuels, platform chemicals \& biorefinery concept. Prog Energy Combust Sci. 2012; 38(4):522-50.
3. Gong F, Li Y. Fixing carbon, unnaturally. Science. 2016;354:830.

4. Claassens NJ. A warm welcome for alternative $\mathrm{CO}_{2}$ fixation pathways in microbial biotechnology. Microb Biotechnol. 2017;10(1):31-4.

5. Schwander T, von BL S, Burgener S, Cortina NS, Erb TJ. A synthetic pathway for the fixation of carbon dioxide in vitro. Science. 2016;354(6314):900.

6. Fei Q, Puri AW, Smith H, Dowe N, Pienkos P. Enhanced biological fixation of methane for microbial lipid production by recombinant Methylomicrobium buryatense. Biotechnol Biofuels. 2018;11(1):129.

7. Müller JE, Meyer F, Litsanov B, Kiefer P, Potthoff E, Heux S, Quax WJ, Wendisch VF, Brautaset T, Portais J, Vorholt JA. Engineering Escherichia coli for methanol conversion. Metab Eng. 2015;28:190-01.

8. Yishai O, Lindner SN, Cruz JGDL, Tenenboim H, Bar-Even A. The formate bioeconomy. Curr Opin Chem Biol. 2016:35:1-9.

9. Sakaguchi K, Kurane R, Murata M. Assimilation of formaldehyde and other C1-compounds by Gliocladium deliquescens and Paecilomyces varioti. Biosci Biotechnol Biochem. 1975;39(9):1695-702.

10. Yang J, Zhang C, Yuan X, Zhang M, Mo X, Tan L, Zhu L, Chen W, Yao M, Hu $B$, Yang S. Metabolic engineering of Methylobacterium extorquens AM1 for the production of butadiene precursor. Microb Cell Factories. 2018;17(1):194.

11. Yang X, Yuan Q, Luo H, Li F, Mao Y, Zhao X, Du J, Li P, Ju X, Zheng Y, Chen $Y$, Liu Y, Jiang $H$, Yao Y, Ma Y. Systematic design and in vitro validation of novel one-carbon assimilation pathways. Metab Eng. 2019;56:142-53.

12. Bareven A, Noor E, Flamholz A, Milo R. Design and analysis of metabolic pathways supporting formatotrophic growth for electricity-dependent cultivation of microbes. Proc Natl Acad Sci USA (BBA)-Bioenergetics. 2013; 1827(8-9):1039-47.

13. Bar-Even A, Noor E, Lewis NE, Milo R. Design and analysis of synthetic carbon fixation pathways. Proc Natl Acad Sci U S A. 2010;107:8889-94.

14. Chen Z, Geng F, Zeng AP. Protein design and engineering of a de novo pathway for microbial production of 1,3-propanediol from glucose. Biotechnol J. 2015;10(2):284-9.

15. Wang C, Ren J, Zhou LB, Li ZD, Chen L, Zeng AP. An Aldolase-catalyzed new metabolic pathway for the assimilation of formaldehyde and methanol to synthesize 2-Keto-4-hydroxybutyrate and 1,3-Propanediol in Escherichia coli. ACS Synth Biol. 2019;8(11):2483-93.

16. Douce R, Bourguignon J, Neuburger M, Rébeillé F. The glycine decarboxylase system: a fascinating complex. Trends Plant Sci. 2001;6(4): $167-76$.

17. Kikuchi G, Motokawa Y, Yoshida T, Hiraga K. Glycine cleavage system: reaction mechanism, physiological significance, and hyperglycinemia. Proc Jpn Acad Ser B-Phys Biol Sci. 2008;84(7):246-63.

18. Guilhaudis L, Simorre JP, Blackledge M, Marion D, Gans P, Neuburger M, Douce R. Combined structural and biochemical analysis of the H-T complex in the glycine decarboxylase cycle: evidence for a destabilization mechanism of the H-protein. Biochemistry. 2000;39(15):4259-66.

19. Rebeille F, Neuburger M, Douce RJBJ. Interaction between glycine decarboxylase, serine hydroxymethyltransferase and tetrahydrofolate polyglutamates in pea leaf mitochondria. Biochem J. 1994;302:223-8.

20. Zhang X, Li M, Xu Y, Ren J, Zeng AP. Quantitative study of H protein lipoylation of the glycine cleavage system and a strategy to increase its activity by co-expression of LpIA. J Biol Eng. 2019;13:32.

21. Kochi H, Kikuchi G. Reactions of glycine synthesis and glycine cleavage catalyzed by extracts of Arthrobacter globiformis grown on glycine. Arch Biochem Biophys. 1969;132(2):359-69.

22. Burgos-Barragan G, Wit N, Meiser J, Dingler F, Pietzke M, Mulderrig L, Pontel L, Rosado I, Brewer T, Cordell R, Monks P, Chang C, Vazquez A, Patel K. Mammals divert endogenous genotoxic formaldehyde into one-carbon metabolism. Nature. 2017;548(7669):549-54.

23. Margaretha JG, Jelena J. 5,10-methylene-tetrahydrofolate dissociates into tetrahydrofolate and formaldehyde at physiological $\mathrm{pH}$ and acidic $\mathrm{pH}$, typical conditions used during sample extraction and LC-MS/MS analysis of biological samples. Biomed Chromatogr. 2014;28(7):1041-2.

24. Hong $Y$, Ren J, Zhang $X$, Wang W, Zeng AP. Quantitative analysis of glycine related metabolic pathways for one-carbon synthetic biology. Curr Opin Biotechnol. 2020;64:70-8.

\section{Publisher's Note}

Springer Nature remains neutral with regard to jurisdictional claims in published maps and institutional affiliations. 